\title{
Pharmacogenomic Testing in the Clinic: When to Begin?
}

\section{Kathryn Blake and John Lima*}

Center for Clinical Pharmacogenomics and Translational Research, Nemours Children's Clinic, Jacksonville, FL, USA

Pharmacogenomics is the study of how genetic inheritance affects an individual's response to drugs and has been an area of study since the 1950s [1-3]. It is expected to yield important advancements in innovative drug development, improved drug safety, improved dose determination, advancements in susceptibility disease screening and targeted therapeutics, personalized vaccines, optimization of the drug discovery and approval process, and resultant decreases in overall cost related to health care [1]. Over 4000 publications are listed in PubMed (http://www.ncbi.nlm.nih.gov/pubmed) which include "pharmacogenetic" or "pharmacogenomic" in the title or abstract and 25 percent of these have been published in the past two years. Despite over 115 drugs being listed on the FDA website that include pharmacogenomic information in the labeling, actual clinical use of pharmacogenomic information is not widespread [4].

Hesitancy for incorporation of pharmacogenomic information into clinical management has been attributed to: unavailability of prospective clinical trial data that demonstrates improved outcomes [5-7], uninformative instructions in the drug labeling to guide decision making [8], lack of pharmacogenomic results at the time of drug prescribing, potential for lack of payer reimbursement, few available CLIA-approved laboratory tests, inefficient use of the medical record for making results available to all caregivers, and simple lack of awareness that pharmacogenomic data exists $[7,9,10]$. These barriers are not insurmountable but cooperation between healthcare stakeholders will be needed. Even so, it has been suggested that the time is ripe for clinical use as risks associated implementing pharmacogenomics is low with the potential for substantial benefits [6].

Stringent criteria have already been introduced to support pharmacogenomic testing of drugs at risk of causing severe adverse drug reactions [11]. These include: identification of (1) medical need defined as a high prevalence of ADR, high prevalence of persons with "at-risk" alleles or low prevalence if the ADR consequences are severe, occurrence of the ADR impacts clinical or quality of life measures or has high associated costs, and lack of available and efficient means to monitor for drug response or ADR; (2) clinical utility defined as evidence is available to support a relationship between the variants and drug response and the assay is predictive for a large portion of the population; and (3) ease of use defined as a readily available reliable assay that has quick turnaround time and is relatively inexpensive; and clinicians can interpret and use the genetic information [11]. These criteria may be difficult to meet for many drugs and do not apply to drugs for which pharmacogenomic testing is used to predict a beneficial response. Of the 115 drugs listed on the FDA website, only seven include pharmacogenetic information in a Boxed Warning section indicating risk of a severe ADR is present. For the remainder, the information is included in the Clinical Pharmacology, Drug Interactions, Indications and Usage, or Precautions sections [4] suggesting a lower risk of potential adverse effects associated with having a particular genetic variant. Thus, in the absence of a drug causing a severe adverse reaction, what criteria should be used to determine if pharmacogenomic testing should be performed?

One perspective is to view clinical pharmacogenomic testing from a non-inferiority perspective rather than as an approach superior to current practice standards [6]. In this context, there is little disadvantage for testing drugs which are widely used and testing may provide useful information in determining treatment and monitoring strategies. Costs for pharmacogenomic testing are expected to be negligible because the cost of genotyping assays are continually decreasing and test results are unlikely to generate follow-up costs unlike testing for disease susceptibility which often leads to additional medical tests and expense [6]. In addition, single panels which contain assays for variants across multiple pharmacogenomic relevant genes are already commercially available. Such a panel would only need to be run a once for an individual and results would be applicable to a large number of drugs anytime they are prescribed for the patient. For example, of the drugs listed on the FDA website [4], over half have biomarkers for the CYP2D6, CYP2D9, or CYP2C19 drug metabolizing enzymes and a single drug metabolism genotyping panel would provide personalized information for all these drugs.

In the absence of biomarker information listed on the FDA website, how can clinicians evaluate pharmacogenomic information that is most likely to influence treatment decisions? The weakness of most pharmacogenomic studies published in the past is that the number of genetic markers screened is limited or the number of participants is small resulting in low statistical power, or findings are not replicated across studies [12]. Efforts to improve the quality of published data resulted in the development of the STrengthening the REporting of Genetic Association Studies (STREGA) guidelines [13] which encourages inclusion of replication data among other criteria. Two recently published studies illustrate excellence in pharmacogenomic association reporting [14,15]. In the first study [14], GWA using 300,000 markers and additional fine-mapping was performed in cases with definite or incipient myopathy and controls, all who were taking simvastatin daily. A noncoding SNP in SLCO1B1 was identified that is in nearly complete linkage disequilibrium with a coding SNP linked to statin metabolism. The risk allele was present in $46 \%$ of cases and $13 \%$ of controls yielding

*Corresponding author: John Lima, Biomedical Research Department, Center for Clinical Pharmacogenomics and Translational Research Nemours Children's Clinic 807 Children's Way, Jacksonville, FL 32207, USA, E-mail: kblake@nemours.org

Received January 23, 2012; Accepted January 24, 2012; Published January 26 , 2012

Citation: Blake K, Lima J (2012) Pharmacogenomic Testing in the Clinic: When to Begin? J Pharmacogenom Pharmacoproteomics 3:e115. doi:10.4172/21530645.1000e115

Copyright: () 2012 Blake K, et al. This is an open-access article distributed under the terms of the Creative Commons Attribution License, which permits unrestricted use, distribution, and reproduction in any medium, provided the original author and source are credited. 
Citation: Blake K, Lima J (2012) Pharmacogenomic Testing in the Clinic: When to Begin? J Pharmacogenom Pharmacoproteomics 3:e115. doi:10.4172/2153-0645.1000e115

Page 2 of 4

an odds ratio of 17 for risk allele homozygotes versus wild-type homozygotes for development of myopathy. Replication was achieved in a similar and larger population of patients using simvastatin. In the other study [15], GWA was performed with over 500,000 markers in patients with asthma using inhaled corticosteroids. This study identified a nonsynonomous marker in GLCCI1 in the screening population associated with lung function response which was replicated in four additional populations. This SNP is in complete LD with the functional SNP as determined by elegant ex vivo experiments. The variant allele was present in about $40 \%$ of the populations and patients who were homozygous had an odds of 2.4 of having no response $(<0 \%$ change in pulmonary function) to inhaled corticosteroids compared to wild-type homozygotes. In a follow-up study, a receiver operator analysis using GLCCI1 in combination with a previously identified SNP in CRHR1 identified high and low responders to inhaled corticosteroids with a predictive performance of $70 \%$ [16].

Both of these studies identified a highly prevalent variant (40\% of the population) that predicted a response to medication. Using these data, genotyping patients for these SNPs will yield beneficial clinical information that can avert adverse effects in simvastatin users or aid in assessing response, in combination with other clinical information, in patients with asthma taking inhaled corticosteroids. Waiting for data from randomized controlled trials to determine if using genomic data improves outcomes may be overly conservative slowing the translation to bedside therapeutics and potentially depriving patients of data needed to ensure efficacy and safety of prescribed medicines [5-7,10]. Randomized controlled trials are expensive with cost estimates of a Phase IIIb trial in 2011 over $\$ 47,000$ per participant $[17,18]$. It has been suggested that these trials should be reserved for testing critical morbidity and mortality outcomes or when affecting a large public sector [6].

Thus, the time has arrived to apply data from appropriately designed and analyzed association studies and implement pharmacogenomic testing in the clinic. For some variants, genotyping will be limited to CLIA-approved laboratories until a kit is commercially available. Waiting for results from prospective genotype stratified trials may needlessly delay optimal treatment decisions and follow-up strategies or expose patients to unnecessary harm.

\section{References}

1. Pharmacogenomics: Human Genome Project Information. U.S. Department of
Energy Genome Programs 2011. http://genomics.energy.gov, 2011. Accessed 1/6/2012, at http://www.ornl.gov/sci/techresources/Human_Genome/medicine/ pharma.shtml.

2. Wang L (2010) Pharmacogenomics: a systems approach. Wiley Interdiscip Rev Syst Biol Med 2: 3-22.

3. Kalow W (2005) Pharmacogenomics: historical perspective and current status Methods Mol Biol 311: 3-15.

4. Table of Pharmacogenomic Biomarkers in Drug Labels. Food and Drug Aministration. Accessed 1/4/2012, at http://www.fda.gov/Drugs/ ScienceResearch/ResearchAreas/Pharmacogenetics/ucm083378

5. Drazen JM (2011) A step toward personalized asthma treatment. N Engl J Med 365: $1245-1246$.

6. Altman RB (2011) Pharmacogenomics: "noninferiority" is sufficient for initia implementation. Clin Pharmacol Ther 89: 348-350.

7. Paugh SW, Stocco G, McCorkle JR, Diouf B, Crews KR, et al. (2011) Cancer pharmacogenomics. Clin Pharmacol Ther 90: 461-466.

8. Hudson KL (2011) Genomics, health care, and society. N Engl J Med 365 1033-1041.

9. Relling MV, Klein TE (2011) CPIC: Clinical Pharmacogenetics Implementation Consortium of the Pharmacogenomics Research Network. Clin Pharmacol Ther 89: 464-467.

10. Mrazek DA, Lerman C (2011) Facilitating clinical implementation of pharmacogenomics. JAMA 306: 304-305.

11. Phillips KA, Veenstra DL, Oren E, Lee JK, Sadee W (2001) Potential role of pharmacogenomics in reducing adverse drug reactions: a systematic review. JAMA 286: 2270-2279.

12. Nakamura $Y(2008)$ Pharmacogenomics and drug toxicity. N Engl J Med 359 856-858.

13. Little J, Higgins JP, loannidis JP, Moher D, Gagnon F, et al. (2009) STrengthening the REporting of Genetic Association Studies (STREGA): an extension of the STROBE statement. PLoS Med 6: e22.

14. Link E, Parish S, Armitage J, Bowman L, Heath S, et al. (2008) SLCO1B1 variants and statin-induced myopathy--a genomewide study. N Engl J Med 359: 789-799.

15. Tantisira KG, Lasky-Su J, Harada M, Murphy A, Litonjua AA, et al. (2011) Genomewide association between GLCCl1 and response to glucocorticoid therapy in asthma. N Engl J Med 365: 1173-1183.

16. McGeachie M, Wu AC, Lima JJ, Peters SP, Irvin CG, et al. (2011) Multitrial, Multigenic Predictive Modeling Of Steroid Treatment Response In Asthmatic Adults Am J Respir Crit Care Med 183: A6183.

17. Clinical Trial Costs Are Rising Rapidly 2011 (Accessed 09/30/2011, at http:// www.pharmalot.com/2011/07/clinical-trial-costs-for-each-patient-roserapidly/.).

18. Phase 3 Clinical Trial Costs Exceed $\$ 26,000$ per Patient. PR Newswire, 2006 (Accessed 09/30/2011, at http://www.lifesciencesworld.com/news/view/11080.) 